\title{
Gradhiva
}

GRADHIV

Revue d'anthropologie et d'histoire des arts

$17 \mid 2013$

L'esthétique du geste technique

\section{Arts de faire, arts de vivre. Chefs-d'œuvre inconnus des compagnons du tour de France}

Arts de faire, arts de vivre. Unknown masterpieces of French Master Artisans

Nicolas Adell

\section{(2) OpenEdition}

1 Journals

Édition électronique

URL : http://journals.openedition.org/gradhiva/2638

DOI : $10.4000 /$ gradhiva.2638

ISSN : 1760-849X

Éditeur

Musée du quai Branly Jacques Chirac

Édition imprimée

Date de publication : 16 mai 2013

Pagination : 118-143

ISBN : 978-2-35744-049-74

ISSN : 0764-8928

Référence électronique

Nicolas Adell, «Arts de faire, arts de vivre. Chefs-d'œuvre inconnus des compagnons du tour de

France », Gradhiva [En ligne], 17 | 2013, mis en ligne le 28 mai 2013, consulté le 10 décembre 2020.

URL : http://journals.openedition.org/gradhiva/2638; DOI : https://doi.org/10.4000/gradhiva.2638

(c) musée du quai Branly 
Arts de faire, arts de vivre

Chefs-d'œuvre inconnus des compagnons du tour de France

par Nicolas Adell

Le chef-d'œuvre, l'ouvrage qu'il faut réaliser pour obtenir le titre de "compagnon du tour de France », est réputé manifester la très haute compétence technique de son exécutant. Et sans doute s'y joue-t-il, parfois, l'expression d'une certaine virtuosité technique. Cependant, l'essentiel est ailleurs. Tous les aspirants au titre de compagnon sont d'excellents ouvriers dont la compétence est déjà reconnue, y compris au sein de la communauté compagnonnique. Aussi la réalisation du chef-d'œuvre vient-elle davantage rendre compte d'une manière de procéder, d'un style, d'une façon de vivre et d'agir son identité compagnonnique que d'un niveau technique. Plus que l'œuvre proprement dite, c'est l'ensemble du travail préparatoire et de la «vie avant l'œuvre » qui constituent le chef-d'œuvre à part entière et font l'objet essentiel de l'examen.

mots clés

compagnonnage, geste

technique, style de vie,

esthétique de l'existence 
1. L'on trouve des organisations de type compagnonnique dans d'autres pays européens, notamment en Allemagne et au Danemark.

2. Concernant le déclin compagnonnique et la politique des regroupements dans la première moitié du $x x^{e}$ siècle, on pourra se reporter à l'excellente synthèse de François Iche (1999: 69-129). Sur l'intérêt dans les années 1930 et 1940 pour le folklore ouvrier en général et compagnonnique en particulier, voir Varagnac 1938, le projet d'exposition au musée des Arts et Traditions populaires lancé en 1939 par Roger Lecotté (1980)

ou encore la documentation rapportée par les acteurs du chantier intellectuel 1810 «Enquête sur l'artisanat rural et urbain » (Trémaud 1962).
Les chefs-d'œuvre des compagnons du tour de France sont connus. Plus exactement, ils sont généralement la part connue d'une communauté que le «profane» ou le «civil » - termes que les compagnons emploient pour désigner ceux qui n'ont pas passé l'initiation compagnonnique - ignore largement quant à d'autres aspects. L'on méconnaît par exemple que le compagnonnage, en France ${ }^{\mathbf{1}}$, ce sont aujourd'hui environ 10000 compagnons (avec quelque 250 à 300 nouveaux reçus chaque année) répartis en plusieurs groupes aux périmètres relativement variables. Depuis les années 1950, trois groupements occupent l'essentiel du paysage compagnonnique français, mettant définitivement un terme à la logique corporative (chaque corps de métier avait alors son compagnonnage, à savoir ses règlements propres, ses rites, son «Devoir», son réseau) qui a présidé sans partage jusqu'à la fin du XIX siècle, laissant désormais la place au principe des «ralliements", des «fédérations», des «alliances", parfois des «fusions". L'Union compagnonnique des Devoirs unis, fondée en 1889, est le témoin encore vivant de cette époque. Elle réunit quelque 1000 compagnons pour une centaine de métiers, ayant élargi aussi loin que possible la définition d'un métier compagnonnique (on y trouve des prothésistes dentaires) et se chargeant d'intégrer, parfois par le biais d'un seul représentant, les métiers d'art (orfèvres, potiers d'étain, etc.) ou ceux en voie de disparition (bourrelier). À ses côtés, l'Association ouvrière des compagnons du Devoir du tour de France, fondée en 1941 à l'initiative des quelques compagnons des «vieux Devoirs» désireux de relancer l'activité compagnonnique alors en déclin et largement folklorisée ${ }^{2}$, réunit aujourd'hui la majorité des compagnons (un peu plus de 5500 pour vingt-cinq métiers) au sein d'un réseau de «maisons» qui couvre parfaitement le territoire métropolitain. Enfin, la Fédération compagnonnique des métiers du bâtiment et autres activités, avec ses 3500 compagnons pour dix métiers, complète le tableau. Créée en 1952 par des compagnons qui ne se reconnaissaient pas dans la nouvelle Association ouvrière qui avait - bien que dans une moindre mesure par rapport à l'Union compagnonnique - ouvert la voie à un certain effacement des spécificités corporatives, la Fédération maintient le principe d'une certaine indépendance des corps de métiers, qui conservent leurs usages et rites compagnonniques spécifiques, ainsi que des sites régionaux. La coutume à Marseille n'est pas exactement celle de Bordeaux, de Paris ou de Tours.

Aussi, en arrière-plan de la tendance aux regroupements, demeurent ancrées dans les esprits et vécues quotidiennement dans l'exercice de la profession l'autonomie et la spécificité de chaque corps de métier. Cela crée un climat de tension relative où, quel que soit le format d'association adopté, la possibilité d'un détachement ou d'une scission plane et devient parfois réalité. Dès lors, le paysage actuel présente une plus grande complexité que l'allure généralement affichée. De l'Association ouvrière s'est détaché en 2000 un groupe de tailleurs de pierre, l'Association des compagnons tailleurs de pierre, dite «l'Alternative», qui possède sa propre organisation, son tour de France, ses coutumes. En 2008, c'est la Société des compagnons selliers, tapissiers, maroquiniers, cordonniers-bottiers des compagnons du Devoir du tour de France qui quitte l'Association ouvrière pour rester «fidèle à son idéal compagnonnique», comme l'explique son président. Enfin, en 2011, quelques compagnons des métiers de bouche de l'Association ouvrière reprennent leur indépendance pour former, 
le jour de la Saint-Honoré, la Fédération des compagnons boulangerspâtissiers restés fidèles au Devoir, qui réunit quelque cent cinquante membres. De même, la Fédération compagnonnique a vu en 2005 un certain nombre de ses charpentiers, couvreurs et plombiers quitter son giron pour former la «Cayenne Itinérante»; et elle a accueilli récemment la Société des compagnons peintres-vitriers du Devoir, un ancien compagnonnage pour lequel nous possédons notamment l'une des toutes premières autobiographies compagnonniques, celle de Jacques-Louis Ménétra ${ }^{3}$ (1738-1812).

Cette nébuleuse compagnonnique n'est composée que pour partie, et en minorité, de compagnons. La plupart des individus rencontrés dans les «sièges», «chambres» ou «cayennes» sont seulement des prétendants au titre. II s'agit de jeunes apprentis en formation initiale qui intègrent un centre de formation d'apprentis (CFA) compagnonnique pour préparer au mieux les épreuves du certificat d'aptitude professionnelle (CAP) et du brevet d'études professionnelles (BEP), et dont quelques-uns seulement poursuivront leur route avec les compagnons. Ils partiront ainsi sur le tour de France en tant qu'«aspirant», «affilié» ou «jeune homme», selon les corps de métiers, pour devenir «compagnon» après quelques années. Les chiffres font défaut mais l'on peut dire que, pour une vingtaine d'entrées en tant qu'apprenti, une seule donne lieu à un parcours complet, l'achèvement du tour de France et l'acquisition du statut de compagnon.

Malgré le morcellement institutionnel du paysage compagnonnique, la disparité des statuts et la pluralité des usages, il existe des points de jonction et des lieux où les discours et les représentations, sinon les pratiques, s'unifient relativement. L'un de ces lieux est la réalisation par les compagnons, à différents moments de leur existence, de chefs-d'œuvre remplissant différentes fonctions. Dans un texte inaugural, essentiellement descriptif, prélude à une étude systématique des chefs-d'œuvre compagnonniques qui reste à faire, Roger Lecotté (1980) dressait une typologie fonctionnelle de ces ouvrages. Aux côtés du «chef-d'œuvre de réception» qui fait accéder à l'état de compagnon et dont il sera plus précisément discuté ici, il identifiait des chefs-d'œuvre de reconnaissance (qui incluent le précédent en quelque sorte), de prestige, de commémoration, de compétition, d'amitié, de retraité, servant d'enseigne (fig. 2), et ceux, plus inclassables en quelque sorte, à savoir les chefs-d'œuvre pour passer le temps, chefs-d'œuvre «violons d'Ingres» (fig. 1). Affinée parfois (Icher 2007: 262-263), cette typologie mérite d'être discutée. Si elle détaille avec soin les différentes fonctions des chefs-d'œuvre compagnonniques, elle ne nous renseigne ni sur ce que ceux-ci signifient ni comment ils signifient. Or il apparaît que le dit essentiel du chef-d'œuvre compagnonnique est l'attachement. II traduit et accompagne l'établissement des liens (sociaux, économiques, affectifs, intellectuels) qui traversent le compagnonnage, que ceux-ci soient hiérarchiques, amicaux ou même hostiles. Se faire la guerre (pour la possession d'un marché du travail par chefs-d'œuvre «de compétition » interposés comme aux XVIII et XIX ${ }^{\mathrm{e}}$ siècles), c'est encore établir un lien. Or l'objet n'y suffit pas en lui-même. Il faut pouvoir y lire l'attachement par les manières et les techniques qui l'ont rendu possible, par le temps qu'il a nécessité, par le degré d'implication de l'ouvrier.
3. Le texte a été déniché, édité et commenté par Daniel Roche (Ménétra 1998 [1982]). 


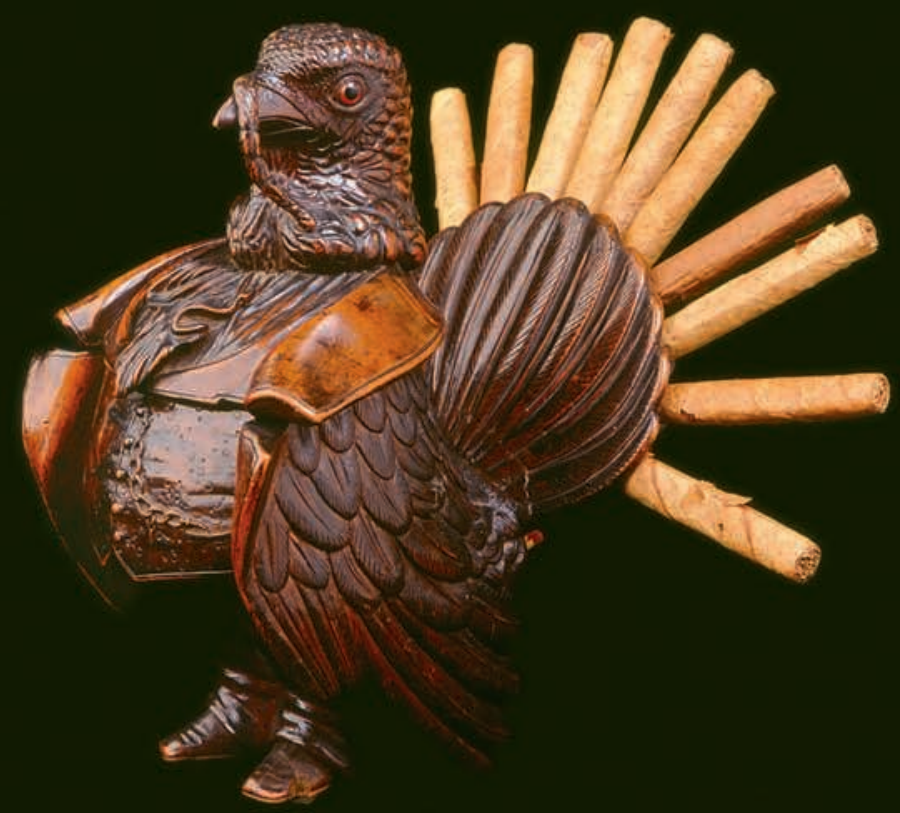

fig. 1

Chef-d'œuvre de

délassement ou "violon d'Ingres " du compagnon forgeron Sylvain Villedieu, 1830-1912, pot à tabac,

porte-cigare et allumettes, XIX siècle. Tours, musée

du Compagnonnage.

(c) Photo Richard Nourry. 
Et les compagnons ne laissent guère d'incertitude sur ce point. Quand il s'est agi, à partir de 2008, de réfléchir à la possibilité d'une candidature collective des groupements compagnonniques pour une inscription sur la liste représentative du patrimoine culturel immatériel de l'humanité, il a fallu proposer la lecture d'un monde commun tenu par un ressort qui procurerait à tous les compagnons un «sentiment d'identité et de continuité», pour reprendre les termes de I'Unesco ${ }^{4}$. Leur sont alors apparus relever fondamentalement de cette catégorie le goût et les manières proprement compagnonniques de transmettre les savoirs. Non le tour de France en général mais ce qui, pendant cette période de formation, se passe dans les ateliers; non la mise en avant des chefs-d'œuvre, mais celle de l'ensemble des processus qui en conditionnent l'élaboration.

Et ce n'est pas seulement la logique de patrimoine immatériel qui a rendu nécessaire la mise en avant des processus, des gestes, des représentations, en lieu et place des objets et des monuments. En réalité, les compagnons éprouvent une certaine méfiance à l'encontre de la qualification profane d'une pièce comme «chef-d'œuvre». Y compris un connaisseur aussi avisé que Lecotté qui, sans doute pour satisfaire aux exigences d'une œuvre de vulgarisation (Lecotté 1980), trahit quelque peu la réalité des usages linguistiques des compagnons ${ }^{5}$. Ces derniers tendent en effet à réserver le terme «chef-d'œuvre" aux réalisations collectives (ou à but collectif), tels ces grands ouvrages destinés à servir d'emblème au corps de métier d'une ville particulière (et que l'on retrouve si fréquemment chez les couvreurs et les charpentiers; fig. 7). Pour les autres travaux, plus individuels, les compagnons préfèrent parler de «travail de réception» (pour devenir compagnon) ou de «maquette», et il n'existe aucun terme correspondant précisément à ces travaux personnels effectués pour le plaisir de l'accomplissement d'un geste ou par amitié. Dans l'ordre des productions individuelles, les chefs-d'œuvre sont ailleurs. Ils désignent notamment ces pièces réalisées pour les concours organisés par la Société des meilleurs ouvriers de France (MOF) auxquels des compagnons participent régulièrement mais qui sont à dissocier du parcours compagnonnique. S'il est permis dans ce cas de parler de chefs-d'œuvre, c'est que, du point de vue compagnonnique, la dimension collective se manifeste au travers du geste individuel. Un compagnon qui se présente au concours MOF le fait, certes, en son nom propre mais il a aussi la charge explicite de défendre et de réaffirmer la valeur de la formation compagnonnique (fig. 3).

Cependant, l'objet lui-même, exposé dans des musées ou dans les sièges compagnonniques, est saisi par un regard qui le qualifie, vraisemblablement imprégné des critères de l'objet d'art occidental: la manifestation d'un savoir-faire non partagé par une majorité, la mise en retrait de toute dimension fonctionnelle et, éventuellement, un moment ou un lieu (une exposition, un musée) qui institue l'art. Les compagnons n'échappent que difficilement à l'emprise de l'impératif “C'est de l'art! ». Aussi ne satisfont-ils pas toujours avec une extrême rigueur aux règles lexicales qu'ils rappellent à l'ethnologue qui les interroge sur ce point: «chef-d'œuvre», pour simplifier, mais aussi parce que le vocabulaire fait défaut dans plusieurs cas de figure (les ouvrages d'amitié et de commémoration notamment).
4. Sur les rapports entre compagnonnage et patrimoine culturel immatériel, je me permets de renvoyer à Adell 2011 et 2012.

5. Inutile de s'appliquer à signaler la liste des textes qui font le "sacrifice» du lexique. L'on peut au contraire souligner ceux qui à l'instar de François Icher (2007) et de Laurent Bastard (2008) s'appliquent à rendre compte, avec la précision que permet un exposé destiné à un public élargi (notamment en étant pointilleux dans les légendes dans le dernier cas), des différentes appellations. 
6. Cas superbe ici, et qu'il faudrait explorer en détail, d'un lieu réunissant l'ensemble des critères du processus d'artification (Heinich et Shapiro 2012) et auquel il est singulièrement opposé une résistance de la part des acteurs.

\section{ci-contre}

fig. 2

Treize scènes de compagnonnage entourant saint Éloi en évêque entouré de deux chiens, 1878 , enseigne de maréchal-ferrant en forme de fer à cheval. Marseille, musée des Civilisations de l'Europe et de la Méditerranée (MuCEM). (C) RMN-Grand Palais (MuCEM)
Cependant, le sacrifice linguistique ne présuppose pas nécessairement celui de la pensée. C'est d'ailleurs ce qui crée le malaise, que j'avais d'abord interprété comme de la méfiance tant les compagnons me reprenaient quand j'employais le mot à mauvais escient alors même qu'ils s'accordaient à eux-mêmes une plus grande liberté. C'est que, sans doute, ils savaient de quoi ils parlaient, en dépit du lexique, alors que l'illusion terminologique devait avoir pour moi, ignorant du réel, l'allure de la réalité. Les compagnons pensaient, à juste titre, que je prenais le "chef-d'œuvre" au pied de la lettre. Tout m'avait en effet porté à croire que nous étions dans le registre de l'œuvre d'art dont les objets compagnonniques, et notamment ces travaux réalisés pour l'acquisition du statut de compagnon, présentaient de nombreuses caractéristiques. De celles-ci, imprécisément formulées d'ailleurs, se dégageait nettement le critère de singularité. Tout «travail de réception ", toute «maquette » est unique. D'ailleurs, il me fut facile de comprendre par la suite que, si la surveillance lexicale des compagnons sur mon discours s'était atténuée, cela ne signifiait ni un relâchement de l'attention ni un renoncement à m'instruire, mais bien l'admission au sein d'un cercle proche d'individus qui tout en n'étant plus des profanes ne peuvent pas pour autant prétendre à une initiation. Cette catégorie de personnes, présente à l'esprit de tous les compagnons quel que soit leur groupement, n'a d'existence linguistique que pour quelques-uns, comme les compagnons charpentiers du Devoir qui qualifient ceux qui en relèvent de «renards éclairés».

En renard éclairé, je saisissais confusément que le malaise des compagnons venait de l'idée d'art, du jugement étroitement esthétique porté sur le chef-d'œuvre qui tend à en faire l'objet inutile par excellence, réalisé dans le confort d'un temps de loisir. II n'en est rien, et tout particulièrement pour le chef-d'œuvre de réception dont il sera ici question. Mais si les compagnons se méfient du jugement esthétique profane et refusent catégoriquement d'être qualifiés d'artistes ${ }^{6}$ (''étiquette étant accusée d'effacer le «travail»), ils n'en arrivent pas cependant à mettre entre parenthèses la dimension esthétique dans la réalisation du travail de réception. Celle-ci est au contraire le centre d'attentions particulières, appliquées toutefois aux gestes (à la situation, au contexte, etc.) plutôt qu'à l'objet réalisé. La langue, une fois encore, est révélatrice. Exécuter le chef-d'œuvre chez les compagnons se dit «tailler la réception". Disparu l'objet, restent l'enjeu initiatique surplombant et, surtout, l'ensemble des actions («tailler» est confus: cela va du traçage à la coupe et à l'assemblage) qui le conditionnent. "Tailler la réception " désigne à la fois un programme technique, une réflexion sur soi et un mode de vie que les compagnons évaluent ensemble aux différents moments de l'opération.

\section{La "critique", ou l'exercice de la faculté de (se) juger}

Il est un moment privilégié pour déterminer les critères du jugement esthétique chez les compagnons: c'est celui où, une fois l'ouvrage achevé, on attend l'heure de l'initiation suspendue à la "critique ", c'est-à-dire à l'examen de l'œuvre du prétendant par plusieurs compagnons qui en soulignent les qualités et les défauts. Ce moment varie fortement d'un groupement à l'autre, voire d'un corps de métier à l'autre. Selon les usages, il peut tendre au dialogue bienveillant, prélude à l'accueil d'un nouveau pair, comme 


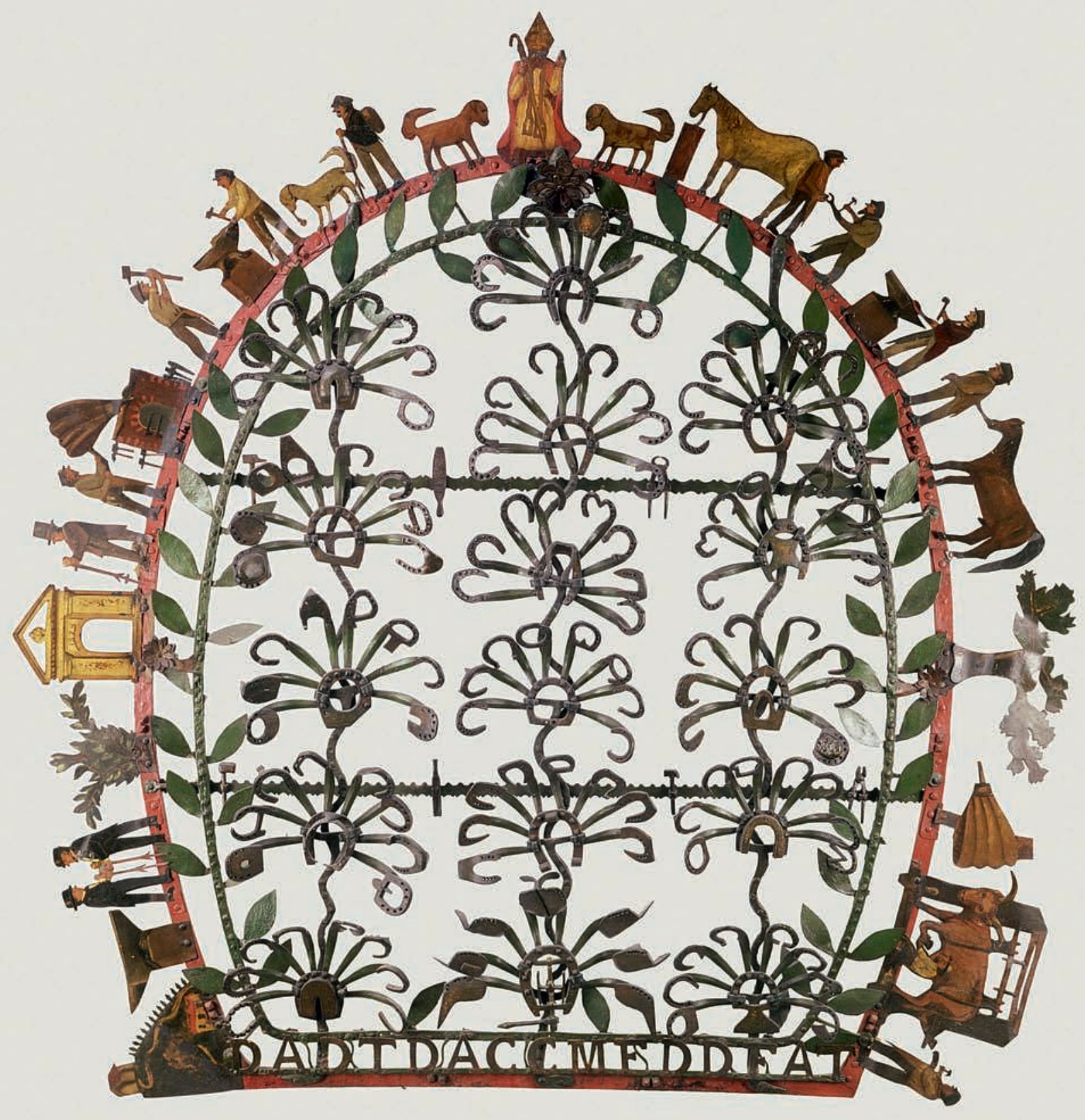


7. Des cas italiens dans Cerutti 2010: 585. souvent à l'Union compagnonnique. Mais dans la mesure où le système de l'Union ne pratique pas la formation initiale au métier et n'accueille donc que des ouvriers déjà formés et souvent même expérimentés, il est vrai que se présentent à la critique des ouvriers d'un certain âge qui inscrivent cette réalisation dans un parcours parfaitement intellectuel et non strictement professionnel. À l'inverse, à l'Association et à la Fédération, le moment de la critique est généralement l'occasion d'une mise à l'épreuve du candidat auquel on ne pardonne aucune de ses insuffisances. La divergence n'est pas récente. On la trouve dans la description des examens professionnels qui se généralisent en Europe au XVIII siècle et auxquels étaient soumis ceux qui souhaitaient accéder à la maîtrise dans le système corporatif d'Ancien Régime (Berg, Hudson et Sonenscher 1983). À cette époque déjà, le critère d'âge en transformait l'allure, les ouvriers expérimentés acceptant de mauvaise grâce (et réclamant souvent des dérogations) l'exigence de produire un chef-d'œuvre et préférant se présenter à un examen simplifié ne portant que sur des connaissances plus théoriques?

La ressemblance de ce moment "critique» avec la soutenance de thèse dans le monde scientifique n'échappe en rien aux compagnons, qui filent tant la métaphore qu'elle devient le signe d'une connivence spécifique. Tout porte à croire en effet, bien que l'on manque d'indications explicites, qu'au moment où la pratique d'un «travail de réception" se réorganise et se systématise dans le compagnonnage (seulement dans le courant du $x x^{e}$ siècle), l'on se serait inspiré du seul grand rite scolaire qui semblait alors équivalent. Sans doute, la présence significative d'intellectuels de formation dans les rangs des groupements compagnonniques des années 1920 aux années 1940 (période d'étiage du vivier compagnonnique) autorise cette porosité des mondes. Celle-ci donne à la «critique » d'aujourd'hui un caractère qu'elle n'avait probablement pas lors des anciens examens corporatifs et qui en fait le moment d'une évaluation plus générale et condensée de l'ensemble des qualités et des défauts de l'aspirant, tant sur le plan technique qu'intellectuel et moral. Le cas des compagnons charpentiers fournira ici le matériau ethnographique nécessaire à l'analyse.

Quand je taillais la réception, je ne savais plus où j'habitais. C'était la folie! On bossait jusqu'à pas d'heure, et on embauchait le lendemain au radar. Le truc, c'est qu'au boulot le singe [le patron] il s'en moquait pas mal que tu tailles pour les compagnons: il fallait que tu sois d'attaque. Et les compagnons, tu savais qu'ils t'attendaient à la fin et qu'ils ne te louperaient pas. (Angoumois l'Ami des Filles)

Les compagnons qui attendent: voilà les traits que prend la «critique" pour l'aspirant qui est sur le tour de France et projette de devenir à son tour compagnon. Cette attente n'est pas seulement supposée ou fantasmée. En effet, si le jeune itinérant se décide à tailler la réception, c'est généralement que des compagnons l'invitent à franchir le pas parce qu'ils le jugent "capables" et, comme le disait déjà le vitrier Ménétra dans son journal, parce qu'il faut «faire une fin» (Ménétra 1998 [1982]: 202). Ce moment survient de façon variable d'un individu à l'autre, même s'il est encadré par des usages propres à chaque métier. Chez les charpentiers, l'on ne peut être "capable» avant trois ou quatre années passées sur le tour de France. Et ne 
pas avoir répondu à cet appel après six années d'itinérance rend l'itinérant suspect: veut-il vraiment s'engager plus avant dans la société compagnonnique? La «capacité», dans le lexique compagnonnique - utilisée à la fois pour qualifier un potentiel technique, une performance chantée en public ou la conduite d'une cérémonie -, n'est pas une propriété que l'on acquiert une fois pour toutes. II appartient à chacun de mettre à profit cette qualification provisoire, nécessaire à la réalisation de tâches spécifiques comme tailler la réception, mener un défilé compagnonnique, chanter ou parler en public, etc. «Puisque les compagnons m'en ont jugé capable»: telle est la formule habituelle qui introduit ces exécutions techniques, rituelles et coutumières.

On est souvent désigné capable officieusement, au détour d'une discussion informelle.

C'est arrivé comme ça. Je venais de débarquer à Anglet. C'était ma quatrième ville sur le tour. Et un jour, un compagnon avec qui j'avais bien accroché me dit: «Faudrait peut-être que tu penses à tailler la réception. » Dans ces cas-là, tu ne dis pas que tu ne te sens pas, que tu n'es pas prêt. Je crois que j'ai dit «Tu crois? » ou un truc dans le genre. II m'a dit «oui, oui », et c'était réglé. Sauf que je savais ce que ça voulait dire. Je les avais vus, les copains qui taillaient la réception; et les compagnons qui étaient sur leur dos toute l'année. Sur le coup, tu es fier parce qu'on te dit que tu es capable, et en même temps je n'étais pas sûr de l'être. (Beauceron la Sincérité)

Il faut alors officialiser ce nouvel état pour lequel il n'est pas de qualificatif spécifique (l'itinérant «taille la réception », voilà ce qui le désigne), et rendre concrète et publique l'attente des compagnons à son égard. L'itinérant sait qu'il doit pour cela écrire, à la main précise-t-on, une lettre dans laquelle il aura à détailler les raisons qui l'amènent à souhaiter être «reçu » par les compagnons. C'est déjà tailler la réception que de décrire ses intentions et procéder à ce retour sur soi. Or, là n'est pas la partie du processus à laquelle les itinérants se plaisent à consacrer leur temps. On remet toujours la lettre à plus tard, ce qui ne manque pas d'ailleurs d'impatienter les compagnons qui expriment alors plus clairement une attente générale: «Tu as fait ta lettre? Tu as fait ta lettre? Ça revenait en permanence. Je n'arrivais déjà pas à m'en sortir avec ma maquette, alors la lettre... Des fois, on en rigolait entre itinérants. On en réveillait un au milieu de la nuit et on gueulait: "Tu as fait ta lettre?" » (Beauceron la Sincérité) Mais à l'approche du printemps, quand le travail doit être achevé (avant la Saint-Joseph, le 19 mars, fêtée par les compagnons charpentiers), il n'est plus possible de reculer. La «critique» est programmée.

Des compagnons qui attendent donc. Qu'attendent-ils exactement? Le chef-d'œuvre, certes, mais pas seulement. II faut l'accompagner d'un environnement, à la fois sensible et intellectuel, dans lequel il prend son sens. C'est cet environnement qui est attendu et demande à être explicité alors qu'il est ordinairement sous-entendu. II y a dans le chef-d'œuvre compagnonnique, et peut-être est-ce aussi ce qui le rapproche de l'œuvre d'art, un travail de condensation, au sens freudien du terme, qui le charge de significations multiples, l'épaissit d'interprétations, le surdétermine dans 


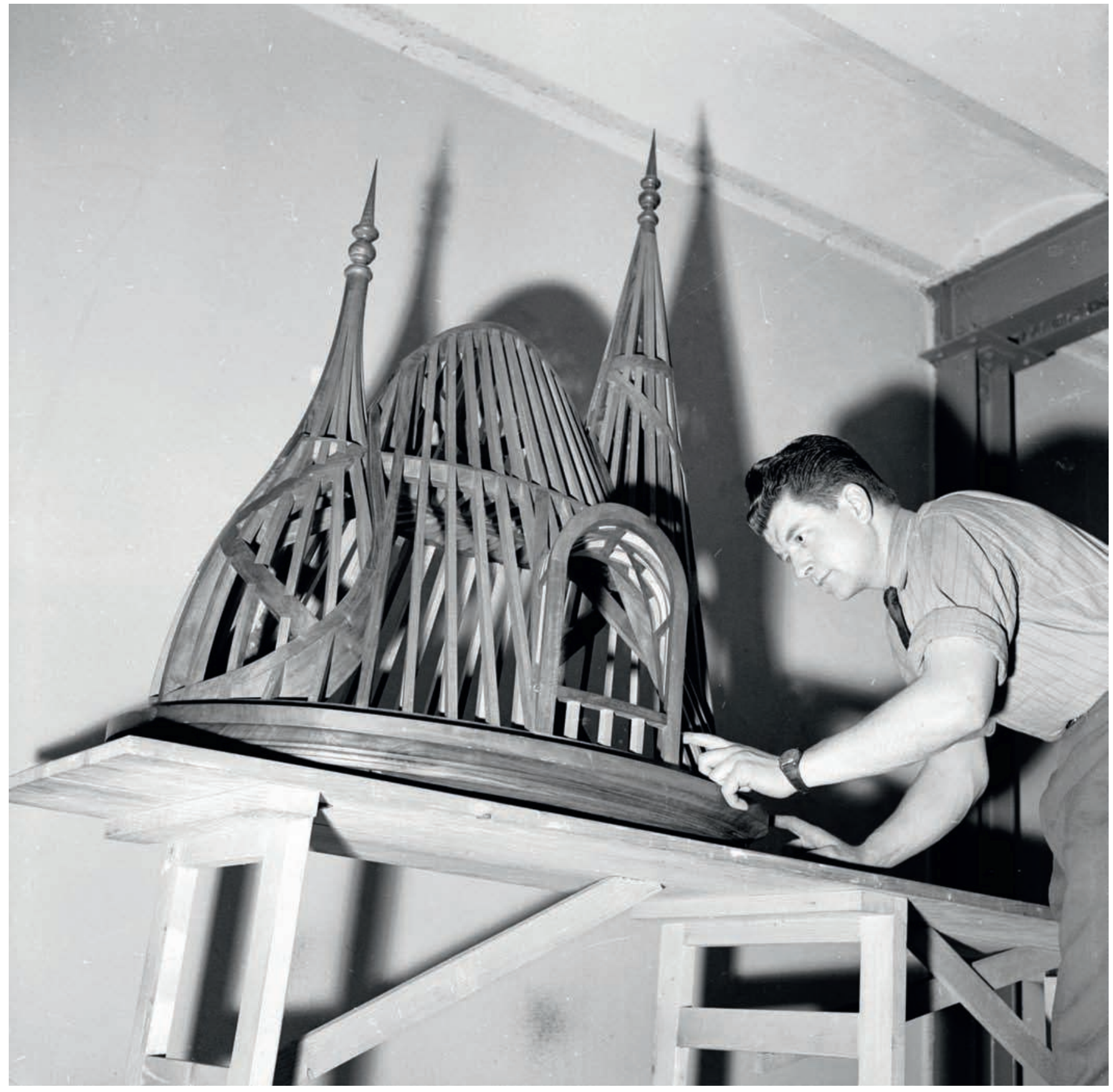

fig. 3

Chef-d'œuvre réalisé par

un compagnon du Devoir,

mai 1956. (o Roger-Viollet. 
des registres très divers ${ }^{8}$. Au moment de la critique, il faut déplier l'œuvre et l'œuvrier. La lettre, remise quelque temps auparavant, dévoile ainsi les intentions, le sens de l'entreprise, et situe l'objet dans un parcours biographique. À ce sens général, qui est presque une direction, s'ajoute l'explicitation d'un sens plus particulier. II est en effet demandé à l'itinérant de présenter son travail, de le soutenir, d'évoquer les traditions techniques par rapport auxquelles il se situe, de faire état, en somme, de la culture qu'il recèle. C'est, outre les difficultés techniques que seul un regard averti peut mesurer, ce qui distingue généralement le travail de réception des maquettes effectuées les années précédentes sur le tour de France. L'ouvrage est ainsi l'occasion d'attester sa connaissance des légendes et de l'histoire compagnonniques, son attachement à la ville dans laquelle on est sur le point de devenir compagnon ou sa maîtrise des traditions du métier (par la connaissance des grandes œuvres qui en ont fait l'histoire, des symboles qui le représentent, etc.). Tels sont les grands registres de la culture manifestés dans le travail de réception: le métier, l'histoire et les traditions locales, le compagnonnage. Plus rarement, le futur reçu y exprime une sensibilité personnelle, son attachement à un loisir, un sport, une pratique (fig. 7). Mais, au-delà du sujet retenu et du référent, l'important est que l'ouvrage signifie et qu'il trouve une raison d'être culturelle. Aussi, quand il arrive, comme souvent d'ailleurs chez les charpentiers, que le travail de réception ne s'affiche pas explicitement comme porteur de culture (les maquettes des charpentiers sont moins «figuratives", à la fois pour des raisons techniques et coutumières, que celles des pâtissiers ou des tailleurs de pierre), le registre technique est alors surinvesti. C'est par exemple le cas de la maquette de réception d'un charpentier bordelais, réalisée en 2001 et exposée dans le petit musée qui jouxte le siège de la Fédération compagnonnique à Bordeaux. Elle n'affiche aucun «trait de culture » lisible par un profane et n'a pas nécessité un nombre d'heures de travail exceptionnel (280 est-il précisé, quand la réalisation d'un travail de réception en charpente en requiert fréquemment 400 ou 500). Aussi le cartel de présentation qui accompagne la pièce doit-il la rendre plus légitime: «La complexité de l'ouvrage réside dans la particularité du tracé (pièces de bois à devers et de forme triangulaire). " Aucune autre maquette du musée ne comporte ce type de mention révélant le référent, le «trait de culture»: la «complexité» dans le cas présent.

L'on rejoint ici un autre aspect, une autre attente des compagnons au moment de la critique: la manifestation de la maîtrise d'une certaine culture technique. II s'agit là de déplier le chef-d'œuvre au sens propre du terme. Dans certains cas, et notamment en menuiserie et en charpente, il pouvait être demandé au candidat de désassembler puis de réassembler son ouvrage sous les yeux des compagnons. Par ailleurs, on exige toujours de l'itinérant qu'il présente l'épure de son œuvre, c'est-à-dire le travail préparatoire de géométrie descriptive que les compagnons appellent «trait 9 » (que I'on retrouve en charpente, en menuiserie ou, sous des formes différentes, en taille de pierre), repassé à l'encre de Chine et qui subit, au même titre que l'ouvrage, l'examen sévère du jury compagnonnique ${ }^{\mathbf{1 0}}$ (fig. 4). C'est à la lecture de l'épure, parfaitement imperméable au profane, que se dévoilent des choix théoriques, des options techniques qui disent l'attachement à une tradition de métier plutôt qu'à une autre. Ainsi, en charpente, il existe
8. Cette proximité entre le «travail du rêve» et le «travail de l'art " a été soulignée par le critique et historien d'art britannique John Walker (1983).

9. II s'agit, schématiquement, de la vue en plan de l'ouvrage à réaliser, vue au moyen de laquelle sont déterminées les façons de tailler les pièces de bois à assembler.

10. Sur le trait des charpentiers et l'ensemble de cette culture technique, on pourra se reporter aux travaux essentiels de François Calame (1995 et 2005).

Pour une mise en perspective de cette pratique dans le champ de significations du compagnonnage, je me permets de renvoyer à Adell (2004 ; 2008: 20-34). 
11. On aura reconnu ici la combinaison qui fait l'artiste pour Claude Lévi-Strauss (1962: 37). différents types de trait (la typologie elle-même faisant l'objet de débats), et opter pour le «trait par rembarrement " plutôt que pour la "sauterelle" ou affirmer l'antériorité et la supériorité de «l'épure au niveau de devers» seront autant d'indications soumises à la critique.

La critique dégage les points d'articulation entre ces éléments attendus (la lettre, l'ouvrage et son étude préparatoire, ainsi qu'un discours qui expose la culture véhiculée par l'objet); elle est l'occasion de conférer une cohérence à l'ensemble. La juxtaposition inédite d'un discours oral sur la culture compagnonnique, I'histoire locale ou les traditions techniques, d'un texte écrit retraçant une histoire personnelle et un projet de vie, et de l'observation de l'œuvre achevée et de l'épure qui en est la projection idéale fait de la critique une occasion idéale de s'exercer à mettre en relation ces éléments. C'est à la couture de l'hétérogène qu'invite cette épreuve, anticipant sur la découverte de nouveaux rapports (sociaux, culturels, symboliques) dont il faudra faire preuve au moment de l'initiation. Les compagnons font prendre conscience du lien étroit qui s'établit entre le «bricoleur» (que l'ouvrage représente et qui porte toujours la trace du rattrapage astucieux nécessité par les aléas d'un matériau inhabituellement résistant, d'un nœud imprévu ou d'une fatigue passagère) et le "savant" (qu'incarne tout ce qui a la charge de "porter la culture": le sujet de l'œuvre, l'épure au trait ${ }^{11}$ ). Mais il y a plus. S'établissent également à ce moment-là les liens entre les actes de sa vie passée, la situation présente, ses avantgestes (les choix et les opérations techniques manifestés dans l'œuvre), et la geste à venir que la lettre annonce et que l'initiation doit confirmer.

Je ne sais pas trop si je peux te raconter tous les détails... Évidemment, je peux te dire qu'on te fait remarquer, soit sur l'épure soit sur la maquette, tout ce qui ne va pas. Tu sais d'avance que c'est ce qui va se passer mais c'est plus fort que toi, tu te mets à douter de tes capacités et tout... Surtout qu'on te ressort tout ce qui s'est passé pendant l'année, à l'atelier quand tu taillais ou le reste du temps. Et que si tel jour tu n'étais pas sorti, ton chevron il serait mieux tombé. Tu vois le genre. (Dauphiné la Constance)

L'examen du travail de réception est l'occasion d'un passage en revue de la vie de l'itinérant. Non seulement, donc, de son année passée à tailler la réception, mais également de sa personnalité générale que les compagnons décryptent ou disent lire dans l'observation minutieuse de l'objet soumis et de l'épure. S'opère là un travail d'unification de l'œuvre et de la vie que l'initiation viendra achever, c'est-à-dire fonder en nature par l'attribution d'un nouveau nom. Mais au moment de la critique, la conjonction a d'abord lieu dans le registre de l'esthétique des formes, et notamment dans celle du geste technique. Les compagnons ne remontent pas en effet sans transition de l'œuvre à l'esprit de son auteur. Ils construisent un discours qui, de proche en proche, irrésistiblement, mène de la pièce à l'artisan en passant par des révélations progressives, dont la principale est celle du geste technique et de sa qualité. Celui-ci constitue le point de passage essentiel à plusieurs titres. D'une part, il articule l'œuvre à la pensée selon une logique que l'itinérant accepte et qui l'emporte, tant il lui paraît clair que la détermination précise de sa gestuelle est lisible dans la confrontation 


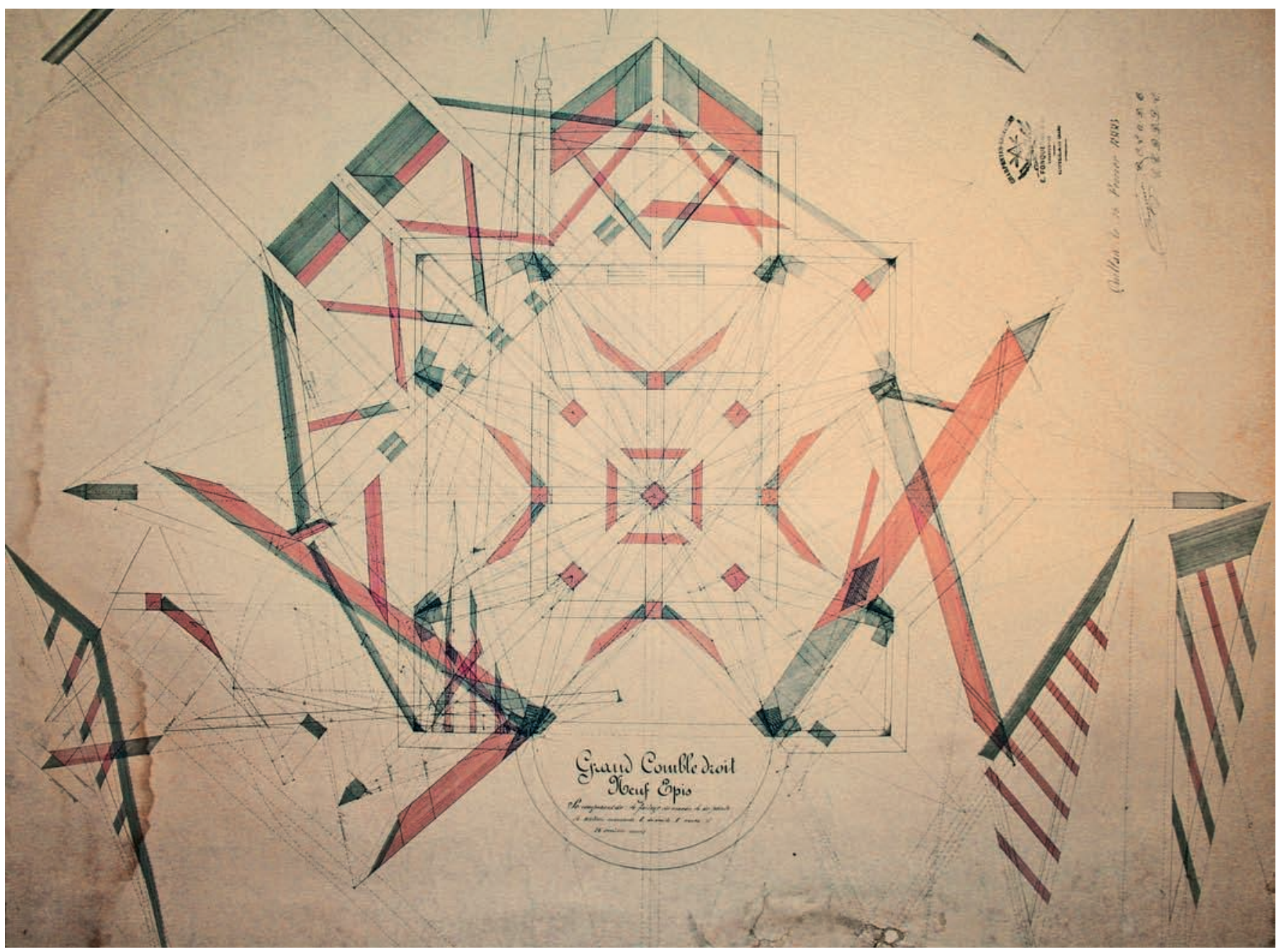

fig. 4

Jean-Paul Chapelle,

La Tradition de l'épure.

Ici, le travail d'un charpentier

de Devoir de Liberté réalisé

en 1888 représentant

un comble. (C) Fédération

compagnonnique des

métiers du bâtiment. 
12. "Jour" : espace qui sépare deux pièces de bois à l'endroit de leur assemblage. Un assemblage idéal ne laisse pas de jour. de la pièce et de l'épure (rendue parfaitement explicite par les opérations de désassemblage et de réassemblage qui peuvent être exigées) et que, du geste technique dès lors retrouvé, il est possible d'inférer les intentions, les projets, les façons de penser le métier. D'autre part, ce passage est renforcé par le discours sur l'unité esthétique des formes de l'œuvre et de la vie. Dans la critique, les compagnons tâchent de démontrer au jeune candidat que sa vie ressemble à ses œuvres, à sa lettre, à son épure. On lui inculque, ou plutôt on lui déclare, la cohérence des styles (techniques, littéraires, mentaux, moraux, culturels) qui «textualisent » les résultats: ce sont eux qui font que la pièce est un «travail de réception», que l'épure est «faite dans les règles», que la lettre révèle «les qualités d'un futur compagnon ».

\section{Tailler et passer la réception}

Cette unité esthétique de la vie compagnonnique, déclarée au moment de la critique, est préparée en amont durant les années de tour de France, mais plus particulièrement durant l'année qui voit le jeune tailler sa réception. Puis elle est renforcée en aval par le rite d'initiation qui ancre définitivement cette conception dans l'esprit des récipiendaires.

Déclaré capable, le jeune itinérant sait que les mois qui suivent vont être difficiles. Pour les charpentiers, ce sont ceux qui s'écoulent entre l'automne, où la capacité est déclarée, et le printemps, où elle doit être attestée par la remise du chef-d'œuvre pour la Saint-Joseph. Quand «tailler» dans cet intervalle? L'aspirant doit en effet concilier la semaine de travail ordinaire chez un employeur et les exigences habituelles du tour de France qui imposent des cours du soir de 20 à 22 heures du lundi au vendredi et des cours pratiques le samedi.

Ben, tu travailles la nuit et le dimanche, quoi! Alors, c'est vrai, au début, tu n'es pas complètement à fond. Tu vas faire un peu la fête ou tu vas te coucher; le dimanche, tu dors. En gros, jusqu'à Noël. Puis là, on te met la pression. $\mathrm{Y}$ a la lettre. Et puis on te dit que tu es en retard: «Quoi? Tu n'as pas fini ton épure? Tu n'as pas commencé à tailler? Je te le dis, mon gars: c'est foutu. " Alors, évidemment, tu mets un grand coup. Tu bosses jusqu'à la Saint-Jo jusqu'à 3 heures du mat' tous les jours et le dimanche. Même les gars qui ont démarré plus tôt ou qui pensaient avoir géré... J'avais un copain qui taillait l'an dernier. Le gars hyper-sérieux. II s'était fait un planning et tout! Un soir, c'était un mois avant la Saint-Jo, un compagnon passe le voir à l'atelier. II taillait. Et il lui fait: «Ça va pas. - Comment ça, ça va pas? - Là, t'as du jour ${ }^{\mathbf{1 2}}$; là, c'est pas droit. - Si, c'est droit! - Je te dis que non. » Et là, le bonhomme, il lui met un petit coup sur sa maquette et lui fait bouger tout le chevronnage! Mon pote, il est à deux doigts de lui mettre sur la gueule. Nous, on est là et on le retient. Le compagnon s'en va: «Tu es en retard» qu'il lui fait en partant! Alors, c'est pas tout le temps comme ça. T'as des compagnons qui viennent te voir, qui te filent un conseil ou qui te donnent un truc. Mais y en a d'autres qui te mettent franchement des bâtons dans les roues. (Angoumois l'Ami des Filles) 
Derrière la brimade ordinaire qui annonce déjà l'étape de l'initiation à la Saint-Joseph, il est un autre enjeu, d'ordre esthétique. En effet, autant qu'une affaire individuelle, tailler la réception est par certains côtés une opération collective. Non qu'elle soit réalisée à plusieurs, chaque ouvrage devant être intégralement, de sa conception à sa fabrication, fait par l'itinérant de manière autonome, ou qu'elle se fasse rigoureusement en public. On peut travailler quand les autres ne sont pas là, profiter d'un moment de solitude pour repasser son épure ou vernir la maquette. Mais il est un rythme général à respecter, auquel «ceux qui taillent » se plient ou sont pliés: l'augmentation continue du temps passé à tailler la réception et les journées sans fin qui caractérisent le mois de février et les premiers jours de mars. Contrairement aux maquettes qui mettent en avant les différences, l'intensité croissante du rythme quotidien de travail unifie véritablement ceux qui taillent et les distingue des autres itinérants. Et les compagnons veillent au respect de la singularité et de l'uniformité de cette cadence, tolérant les disparités individuelles à condition que le tempo idéal de l'année de réception soit conservé. À la fin, tout le monde doit être en retard; il est impensable en effet d'arriver à la Saint-Joseph en coulant des jours sereins, terminant paisiblement, et dans les délais, son ouvrage. C'est d'ailleurs cette accélération imposée qui accentue, paradoxalement, l'importance accordée à l'esthétique du geste technique dans la réalisation de ce travail. Dans la précipitation, l'accomplissement du «bon geste» nécessite en effet une attention et un effort supplémentaires dont les compagnons, par leur présence dans les ateliers, tiennent à s'assurer. On assiste ici à une manifestation esthétique d'un certain type, que je qualifierai de romantique. L'effet recherché est produit par le doux rayonnement d'un geste maîtrisé au milieu de l'affolement des sens et des événements. Mais quels seront dans ces conditions les critères du «bon geste»? Ils sont de deux ordres, intellectuel et sensible.

Intellectuel, d'abord. Le bon geste est donc celui dont l'éclat ressort particulièrement quand, à sa place, on pourrait avoir un geste précipité. Il est le geste guidé par un savoir pesé; et pour le chef-d'œuvre, par un savoir traditionnel. Chez les compagnons charpentiers, tout l'enjeu de la réalisation des ouvrages est de trouver les coupes ${ }^{\mathbf{1 3}}$ qui, si elles sont bien déterminées, permettront l'assemblage idéal qui ne laisse aucun «jour». Or plusieurs méthodes existent pour obtenir les «bonnes coupes» sans que toutes relèvent des «bons gestes»: le perchage, la trigonométrie et le trait. Le «perchage», ou la coupe «à la perche», est la manière sauvage de trouver une coupe. L'on superpose les deux pièces que l'on cherche à assembler et l'on trace sur l'une d'elles la section à opérer, ou bien l'on aligne les pièces dont on veut obtenir la même allure (un chevronnage par exemple), et on les passe ensemble à la scie et au rabot. Entièrement empirique, c'est la méthode la plus dénigrée par les compagnons, et la plus sévèrement condamnée pour la réalisation des maquettes car elle est sans savoir. Or c'est celle par laquelle on est tenté quand le rythme s'accélère.

Une année, j'étais tellement à la bourre pour ma maquette que j'ai posé mes chevrons un peu au pif et là, personne dans l'atelier, paf, je t'ai mis un coup de rabot sur l'ensemble et c'était impec! Bon, je taillais pas la réception. Parce que, quand t'as l'œil, ça se voit quand même: c'est le même sens de coupe, et tout.
13. Par «trouver les coupes", il faut comprendre le fait de déterminer l'angle et la manière de tailler une pièce de bois. 


\section{2 mains}

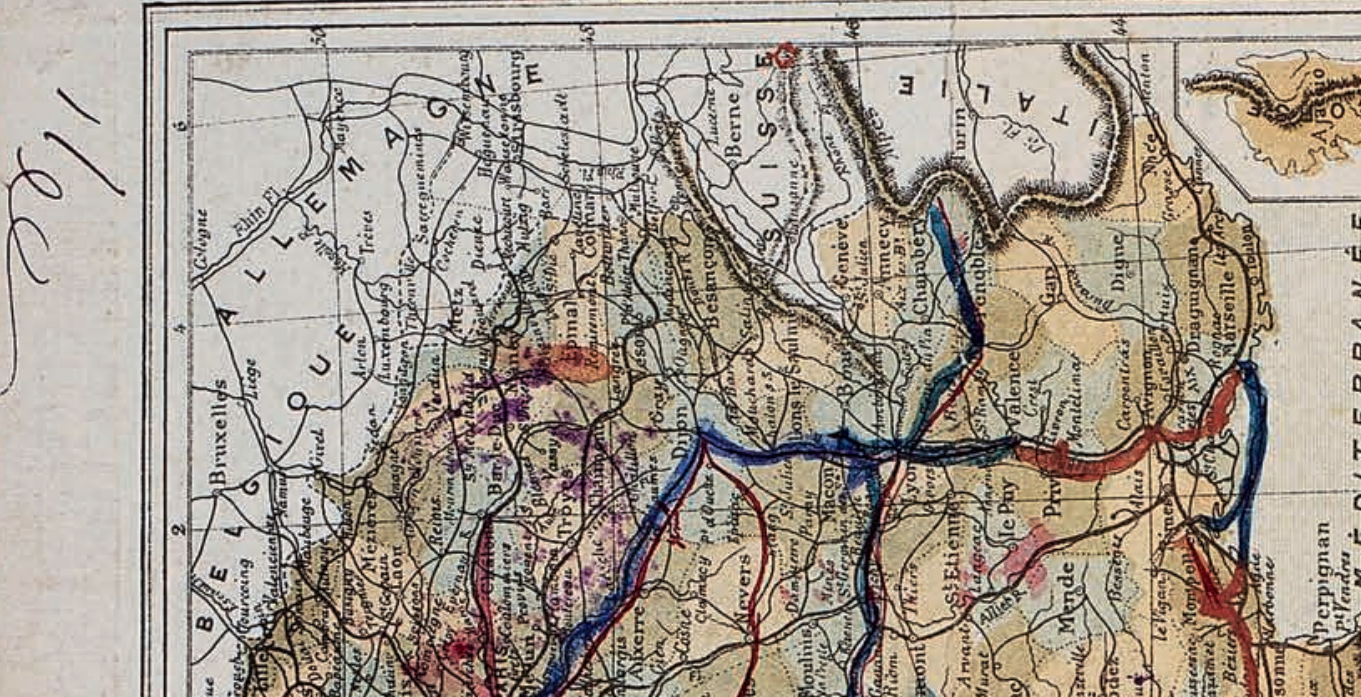

101 (5)

3.0.5.

5.

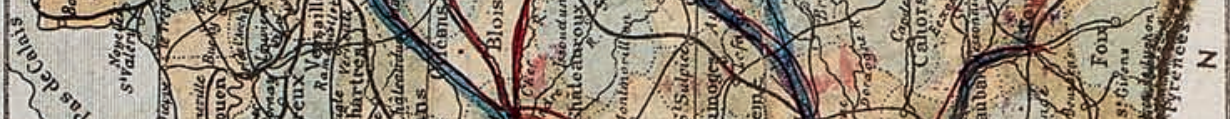

$3 \%$ के

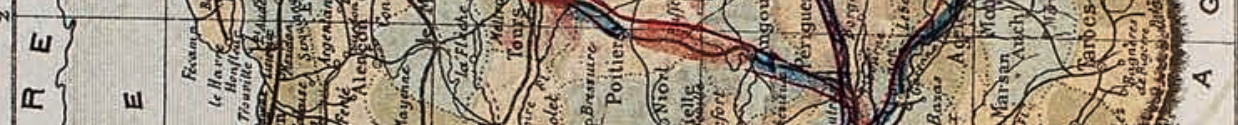

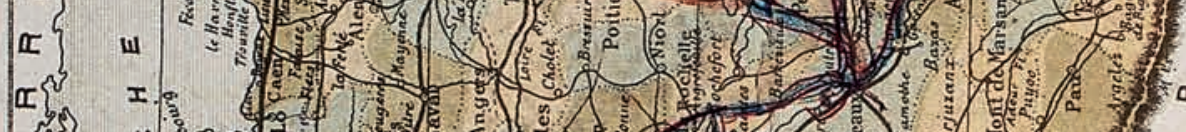

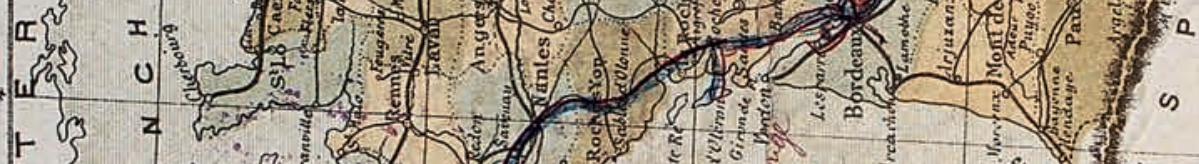

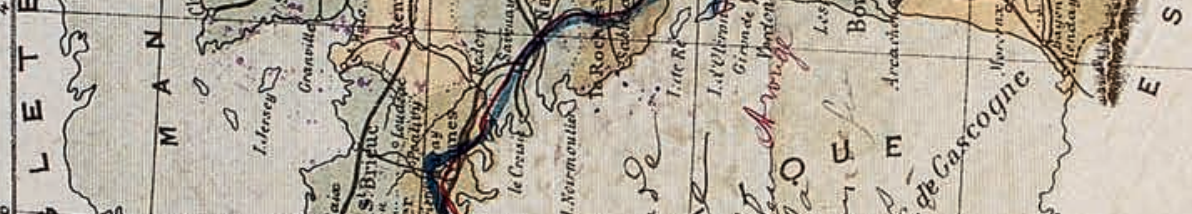

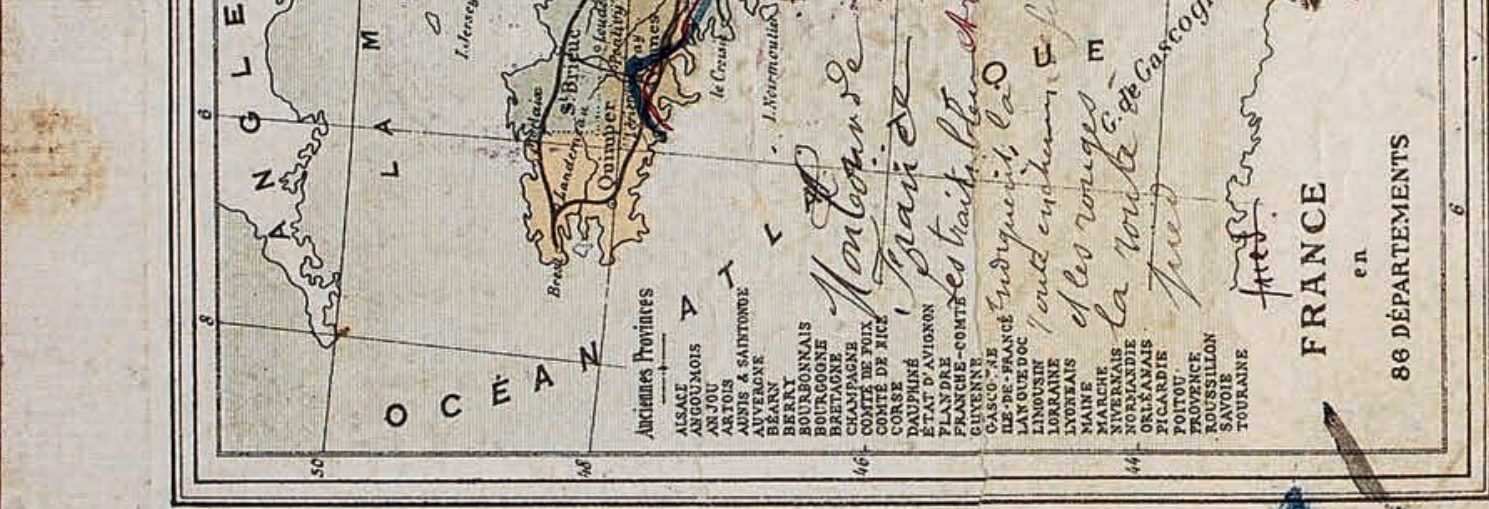




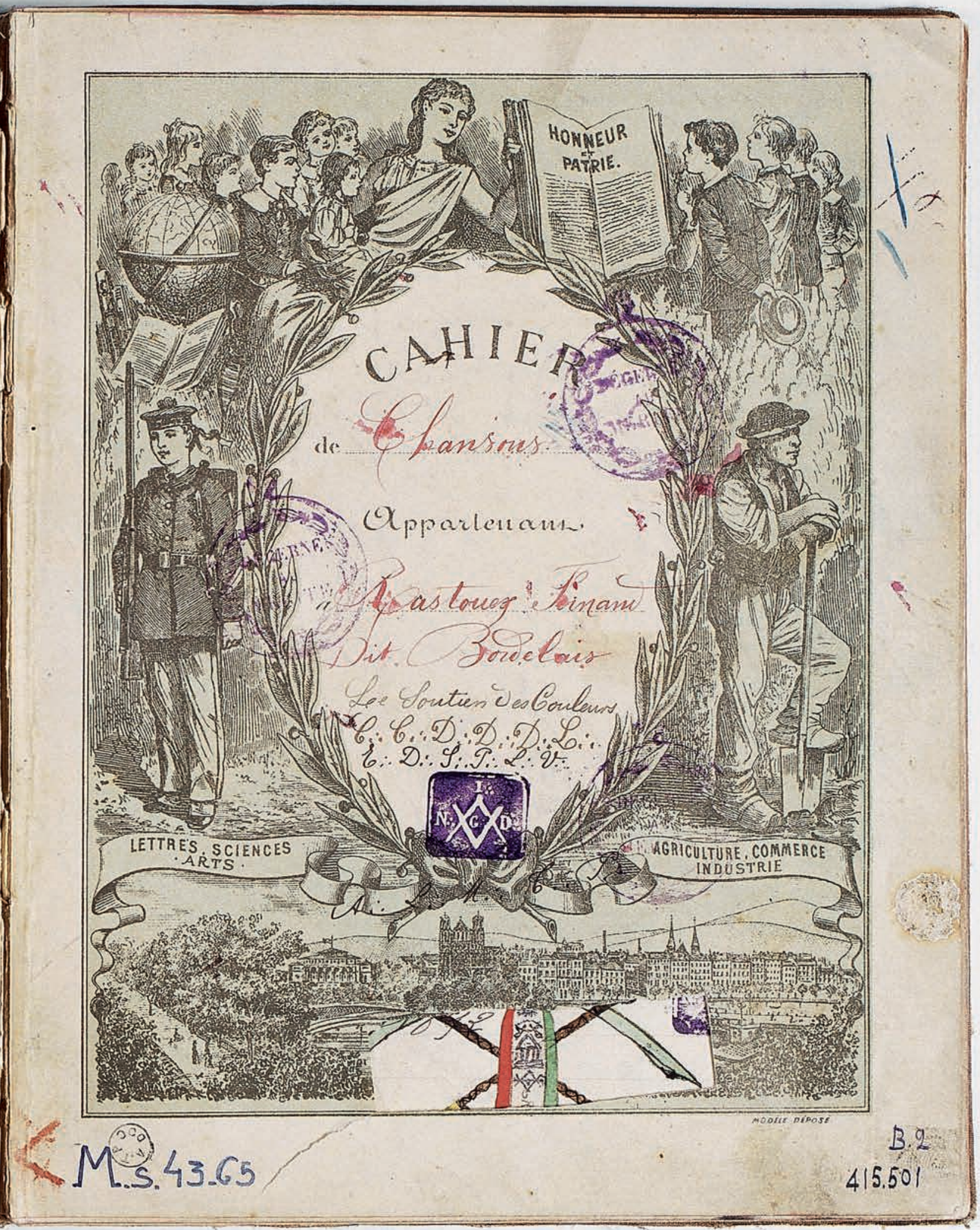


14. L'association

de la musique et du travail est un thème classique de l'anthropologie. Pour une perspective récente et originale à partir d'une ethnographie du travail chez les Sénoufo, lire Lemaire (2009: 102-104 et 111-116 notamment, sur la question des rythmes) et son article dans ce numéro.

15. Pour une approche des fonctions de ces chansons de compagnons, je me permets de renvoyer à Adell 2010: 215-220. Les ethnomusicologues ne s'y sont guère intéressés jusqu'à présent. La thèse en cours de Julie Hyvert devrait combler cette lacune.

\section{double page précédente}

fig. 5

Page d'un cahier de

chansons compagnonniques d'un compagnon charpentier, 1893. Marseille, musée des Civilisations de l'Europe et de la Méditerranée (MuCEM). (C) RMN-Grand Palais (MuCEM)/Hervé Jézequel.
Là, je me suis pas fait prendre parce qu'on te regarde pas la maquette de base comme on te regarde la maquette de réception. (Picard la Franchise)

Mais c'est une méthode qui a ses limites, ne s'appliquant guère qu'aux coupes droites dont un travail de réception ne peut se contenter. À l'opposé du perchage, la trigonométrie permet, par le calcul abstrait pour celui qui le maîtrise, d'obtenir les angles et les sections, y compris courbes. Moins dévalorisée que la précédente, peut-être parce qu'elle est moins communément sollicitée, en particulier dans l'urgence, elle n'en constitue pas moins une méthode condamnable car, quoique avec savoir, elle est sans geste en quelque sorte. II faut en effet la combinaison d'un geste épaissi d'une connaissance dont la maîtrise est attestée par la production du dessin théorique, l'épure, qui montre le savoir du trait permettant, à l'aide de notions géométriques de base, de déterminer par le compas et l'équerre toutes les coupes possibles. C'est cette science (ou cet art, les compagnons sont hésitants sur le qualificatif) que le travail de réception doit manifester, dont la dimension esthétique n'échappe à personne quand, dans le chaos d'un retard généralisé, l'itinérant prolonge sur son épure posée sur la table à dessin l'étude au compas qui lui fait espérer que lors de la critique la traque des jours sera sans résultat. Ce n'est jamais le cas.

Le geste ralenti et maîtrisé par l'application du trait, y compris dans les derniers moments, confère au travail de réception cette dimension supplémentaire, esthétique, qui a la vertu de signifier que le référent est ailleurs que dans la recherche de l'efficacité technique ou dans la seule réalisation de l'objet. II est aussi et d'abord question de maîtrise de soi. Une maîtrise de soi, parallèle et connectée à la dimension intellectuelle, qui est offerte à la lecture des autres itinérants et des compagnons présents dans l'atelier de manière parfaitement sensible. Un geste maîtrisé se voit, mais surtout il s'entend. C'est un geste «musiqué» qui, face au tempo accéléré et de plus en plus chaotique de l'élaboration générale de l'œuvre, assure le maintien d'un équilibre et d'une régularité ${ }^{14}$. Non que les aspirants, dans l'atelier, s'appliquent à travailler collectivement au même rythme, et encore moins à chanter pour cadencer le travail - bien qu'il existe une forte tradition du chant compagnonnique, plutôt d'ailleurs comme chant de cérémonie que comme chant de travail ${ }^{15}$. Mais un soin tout personnel est apporté à l'exercice rythmé du travail dont la présence sonore occasionnelle d'un novice dans l'atelier est un contre-exemple éloquent. Chacun étant à l'ouvrage, être apprenti s'entend en effet avant de se voir. La fréquence exagérée et dysharmonique des coups de marteau, l'insistance un peu trop marquée d'un engin électrique, la durée d'une coupe, etc.: tout bruit d'atelier devient un véritable son à l'oreille du professionnel à même d'interpréter (selon le rythme avec lequel il est reproduit mais également, et plus finement, selon sa hauteur,son timbre, son intensité) à la fois la nature du travail et la qualité du travailleur. Ce n'est pas un hasard si l'origine de la musique était située par les anciens dans les ateliers, notamment les forges, les notes se dégageant de la percussion des outils mêmes (Heller-Roazen 2010). Et si l'idéal mythique d'une harmonie sonore générale de l'atelier n'est jamais atteint, reste aujourd'hui l'importance d'une gestuelle musiquée du métier par un individu dont l'empreinte sonore annonce le savoir et 


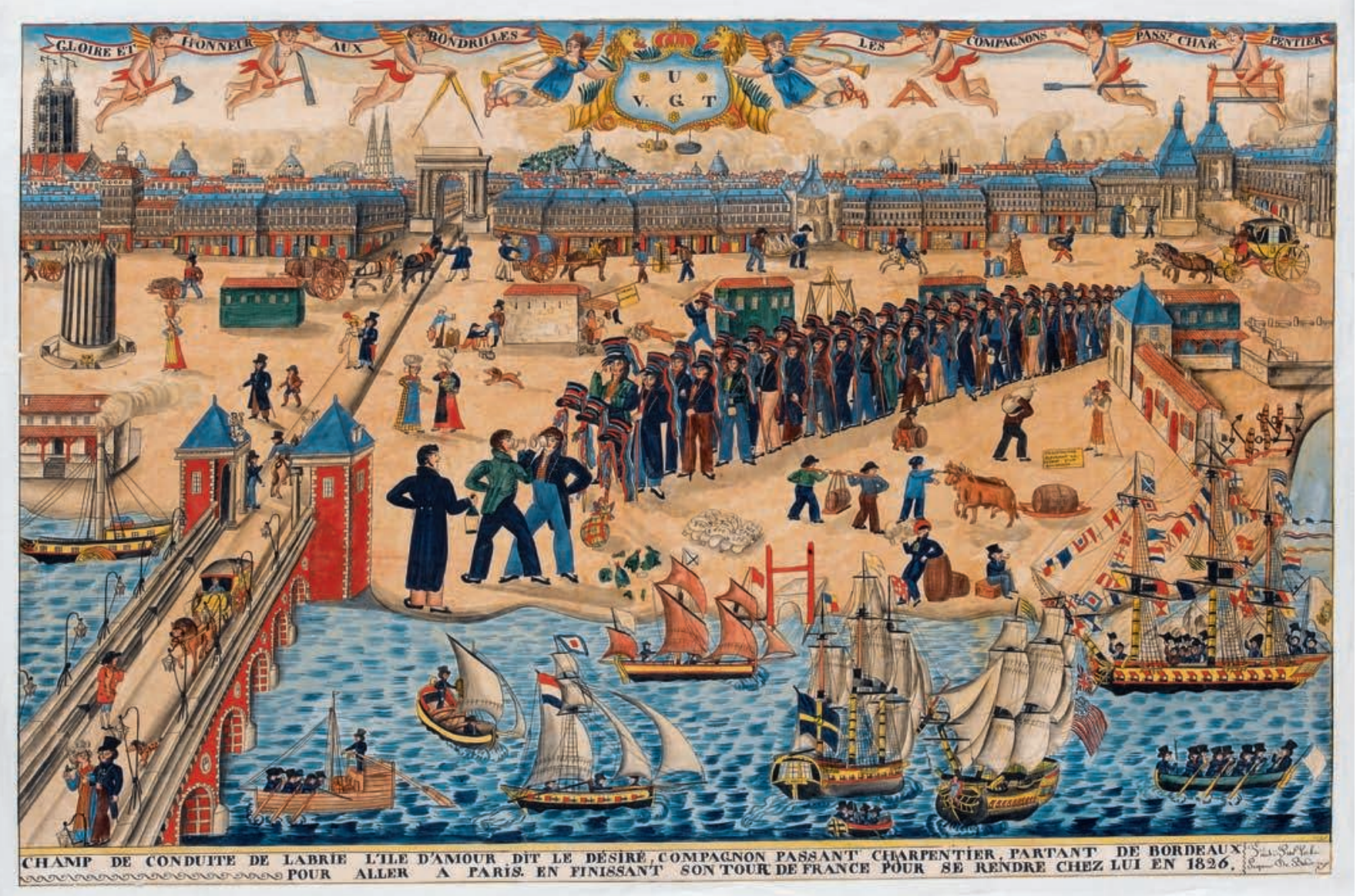

permet, dans l'intensité bruyante de la situation de travail, de discerner le scintillement audible d'une mesure, tout comme l'usage du trait dans l'urgence de l'œuvre laissait deviner une maîtrise intellectuelle.

Ces éclats de gestes techniques esthétisés par la situation dans laquelle ils sont réalisés ont un écho, par-delà la critique qui les coud ensemble et les met en relation avec l'ensemble des dimensions de la personne, dans le rituel d'initiation auquel l'exécution du chef-d'œuvre donne accès. Ce rite, la «réception», concentre la fonction de la critique et les effets recherchés dans les dernières semaines passées en atelier à tailler sa maquette. À la différence de ces moments ethnographiables ou racontables, la réception est chargée d'un secret qui en garantit, selon les compagnons, l'efficacité. Cet aspect emporte sans doute la conviction de tous puisque les trahisons du secret de la réception sont rares dans l'histoire du compagnonnage, se concentrant pour l'essentiel dans la seconde moitié du XIX siècle. Aussi le dernier état d'un rituel de réception rendu public dont nous disposons date des années 1890. II concerne justement les charpentiers et est le fait d'un certain Jean Connay (faut-il préciser qu'il s'agit d'un pseudonyme?). Mais cette trahison pose en elle-même des problèmes d'accès à la réalité du rituel. Connay (1909) veut se venger des sévices subis durant l'initiation et «ruiner le compagnonnage» (avec l'aide des nouveaux syndicats qui voient dans la formule compagnonnique une fig. 6

Champ de conduite de Labrie l'île d'amour, dit le Désiré, compagnon charpentier partant de Bordeaux pour aller à Paris en finissant son tour de France pour rentrer chez lui en 1826. Marseille, musée des Civilisations de l'Europe et de la Méditerranée (MuCEM). ( ) RMN-Grand Palais (MuCEM)/Gérard Blot. 
16. Une description et une analyse détaillées de ce rituel dans Adell 2008: 162-188. sorte de concurrence obsolète). Signe encore de la conviction que révéler le secret de l'initiation menaçait directement l'ensemble de l'institution. L'on ne saurait cependant décider ce qui, dans la révélation extrêmement détaillée du charpentier, relève de l'invention ou du vécu 16. "C'est l'ancien temps", disent aujourd'hui les compagnons charpentiers; "ça, c'est les charpentiers ", disent ceux des autres corps de métiers. Toujours est-il que, dans les années 1980, les charpentiers brûlaient encore cérémoniellement les exemplaires de Connay quand ceux-ci tombaient entre leurs mains. Indice qu'il y a une certaine vérité du récit du traître qu'un recoupement avec d'autres récits de réception, venant de corps de métiers différents, confirme.

Cela offre l'occasion de présenter un canevas du rituel à un niveau de généralité suffisant pour que le profane s'en fasse une représentation mais sans que, dans le même temps, le jeune aspirant charpentier, lecteur éventuel de ces lignes, perde le bénéfice de l'expérience à venir.

Connay décrit un rituel de neuf jours et neuf nuits. II est très probable que déjà à son époque la tendance fût au raccourcissement. Trois jours étaient devenus un standard. Aujourd'hui, le temps d'une nuit est vraisemblablement suffisant. Le récipiendaire est introduit dans une pièce du siège compagnonnique qu'il a d'ailleurs pu fréquenter en d'autres occasions (fêtes qui rythment les départs et les arrivées sur le tour de France, fêtes patronales, réunions de société ou de corps de métier, etc.), mais qui va prendre à ce moment une autre allure. Un décor est installé tandis que des détails déjà là (la présence ordinairement inaperçue d'un symbole sur un mur, au sol ou au plafond) seront mobilisés dans le cadre du rituel, qui consiste en premier lieu à rejouer le voyage (le tour de France) et ses difficultés (alors physiquement éprouvées: le jeune homme est chahuté, bousculé, etc.). Vient alors le moment de manifester son attachement à la communauté, ce qui peut prendre plusieurs formes: la prestation d'un serment ou le dépouillement entier de l'individu (on aura pu lui demander de venir avec ce qu'il a de plus précieux et lui imposer de tout donner à la société ou de tout détruire). Souvent, cette épreuve a la valeur d'un test: pour défendre le compagnonnage, serait-on prêt à renier père et mère, à blesser quelqu'un ou pire encore? Les formules sont rhétoriques et l'on n'attend rien d'autre que des «non»; mais les compagnons sont de bons acteurs et savent insinuer le doute. Dans le même temps, le récipiendaire, dont on a saturé les sens par différents moyens (heurté, privé de la vue puis ébloui, muré dans le silence d'où jaillit un grand bruit et vice versa, etc.), se sent clairement assujetti à l'ordre créé par le rythme régulier et la symétrie du jeu réglé des questions et des réponses auquel il se livre avec le maître de la cérémonie. Enfin, une fois la valeur morale du néophyte vérifiée et la qualité de ses réponses estimée, on lui révèle le sens caché de certains mots, objets ou symboles. Et on lui fait voir le «Devoir », le livre où sont consignées les règles qui président à l'organisation de la vie communautaire, depuis les façons de vivre ensemble jusqu'au déroulement des initiations. Par ces révélations successives, le monde prend un sens supplémentaire pour s'ordonner mieux et à nouveau.

Le rite propose également l'achèvement de l'unification de la personne commencée lors de la critique. L'homme de métier et sa compétence, le compagnon neuf et son attachement à la communauté, l'individu 


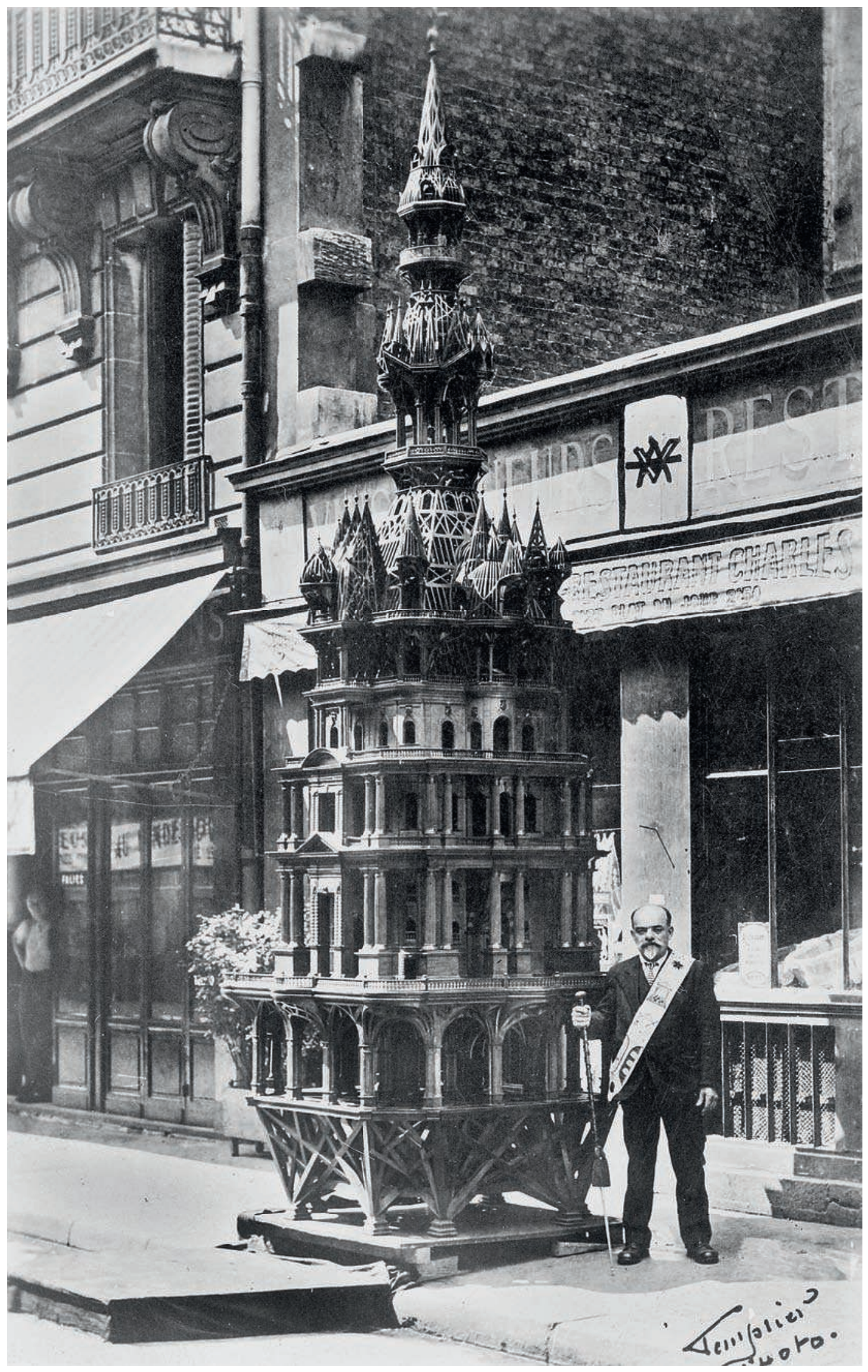

fig. 7

Chef-d'œuvre emblématique de la confrérie Compagnons charpentiers du Devoir de Liberté, Paris, restaurant Les Charpentiers, vers 1900. () Albert Harlingue/ Roger-Viollet. 
17. On théâtralise l'imposition du nouveau nom durant le rituel quand, semble-t-il, celui-ci fait l'objet en amont d'une concertation et d'une négociation entre le néophyte et les compagnons qui s'apprêtent à le "recevoir ». II faut que le futur compagnon s'estime "capable» d'assumer son nom.

18. La référence incontournable sur cette notion dans le monde ouvrier reste Edward P. Thompson (1988 [1963]). et ses centres d'intérêt personnels, ses qualités et ses défauts se rejoignent dans le nouveau nom attribué à l'issue de la cérémonie ${ }^{17}$ : Vendéen le Soutien du Devoir, Limousin la Franchise, Dauphiné la Persévérance, Parisien l'Ami des Arts soulignent un trait et annoncent, pour soi et pour les autres, le programme et le spectacle d'une conduite, basse continue de l'ensemble des registres d'existence de la personne. Aussi, sur le plan éthique, la ligne qu'impose le nom compagnonnique remplit la même fonction que le geste technique exécuté de manière esthétique: s'offrir à la reproduction non seulement du fait de la désignation sociale - le jeune apprend le «bon geste» ainsi qualifié dans l'atelier comme il apprend la constance auprès du compagnon la Constance ou la fermeté auprès de la Fermeté -, mais également en raison des vertus propres de l'imitabilité que contiennent un acte rythmé, symétrique, ou un comportement prédictible (mon nom, c'est moi) au milieu de l'embrouillamini ordinaire des événements de la vie sociale.

\section{Raisons esthétiques: de l'éthique à la technique}

Une question reste en suspens. II n'y avait pas de raison a priori à ce que les compagnons reconduisent l'usage de l'examen professionnel et la pratique du chef-d'œuvre tels qu'on les trouvait dans le système corporatif d'Ancien Régime et contre la rigidité duquel ils s'étaient élevés. Le compagnonnage était alors le moyen de faire sa jeunesse dans le monde artisanal. Y retrouver l'un des leviers de l'ordre hiérarchique, le chef-d'œuvre qui donnait accès à la maîtrise, peut sembler incongru. D'ailleurs, cet usage n'était guère de mise chez les compagnons jusqu'au XIX ${ }^{e}$ siècle, moment où, à des rythmes divers selon les corps de métiers, un chef-d'œuvre commença d'être exigé pour accéder au titre de compagnon. Mais la véritable systématisation de cette pratique n'intervient qu'au $x x^{e}$ siècle. Comment expliquer cette invention, ou plus exactement ce réemploi?

D'une part, l'effondrement du système corporatif, associé aux transformations considérables du monde ouvrier entre la fin du XVIII ${ }^{\mathrm{e}}$ siècle et la première moitié du XIX siècle, avait éloigné assez rapidement du paysage mental des compagnons le référent total que représentait la corporation d'Ancien Régime. L'on pouvait, dans les années 1830, parler à nouveau de "chef-d'œuvre» sans être suspecté de se rallier aux pratiques inégalitaires que cela impliquait un demi-siècle plus tôt. Mieux, à mesure que les transformations du travail se radicalisaient, le chef-d'œuvre pouvait signifier de façon plus univoque à la fois le passé, l'authenticité et l'ancienneté sans en référer à la pesanteur du système social qui régnait à ces époques imprécises. D'autre part, et cela est parfaitement corrélé, cette même période connaît une profonde mutation de l'«économie morale» des compagnons ${ }^{\mathbf{1 8}}$. Institution de passage pour «faire la jeunesse», le compagnonnage devient en effet une institution à rites de passage pour encadrer la totalité de la vie artisanale. Un encadrement qui se distinguait par la mise en œuvre d'un système de valeurs neuf (la chevalerie du travail, I'honneur de l'ouvrier, le Devoir, etc.) que les individus incarnaient par des arts de vivre dont l'apprentissage se faisait durant les années de tour de France, au sein de la vie en communauté et lors des rites initiatiques.

Mais ces exigences morales ou comportementales (la vie commune, le principe hiérarchique, le passage par une initiation, etc.) pouvaient paraître 
exorbitantes. Les convertir en des exigences techniques en renouant avec la pratique du chef-d'œuvre pouvait les rendre acceptables et, surtout, sensibles: reliées, la fabrication de l'ouvrage et la construction de soi allaient de pair. La critique le rappelle aujourd'hui. Mais la nouvelle systématisation du chef-d'œuvre a aussi permis de rendre plus concret un rythme propre à la vie compagnonnique. II s'agit de ce rythme de vie spécifique du moment de sa réalisation, quand on «taille», mais plus généralement de celui d'une vie sur le tour de France scandée par les œuvres réalisées, des petits travaux exigés chaque année jusqu'au travail de réception.

Edward Tylor (1896: 182-228) appelait arts of life l'ensemble des techniques et de la culture matérielle d'une société en tant qu'elles permettent le maintien et la reproduction de la vie concrète. L'expression est superbe et il faudrait l'étendre à l'ensemble des formes immatérielles (aux gestes, aux modèles, aux styles, aux valeurs) qui assurent la même reproduction aux plans cognitif et affectif et qui, en compagnonnage, sont matérialisées par l'ouvrage fait pour la réception.

Sur ces plans résident véritablement, par-delà les maquettes et leur complexité technique, les chefs-d'œuvre inconnus des compagnons qui sont un répertoire de gestes, de formes et de styles de vie cousus dans la trame de l'œuvre et de sa réalisation. Le profane et le jeune itinérant n'y voient généralement qu'un «mur de techniques» (comme, dans la nouvelle de Balzac, Poussin ne se heurtait d'abord qu'à un «mur de couleurs» dans le tableau du maître Frenhofer). Mais, au futur compagnon, apparaît à certains moments la beauté d'un geste réglé ou le détail parfait d'un programme de vie (le nom), le «pied extraordinaire» que Poussin a remarqué dans l'œuvre du maître.

Université de Toulouse II - Le Mirail LISST - Centre d'anthropologie sociale nicolasadell@yahoo.fr 


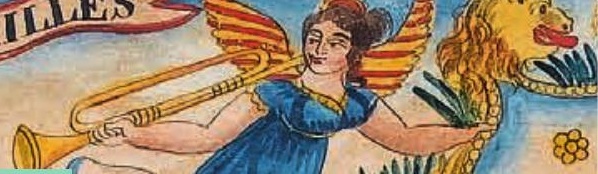

Bibliographie

Arts de faire, arts de vivre. Par Nicolas Adell

\section{Adell, Nicolas}

2004 "Les sentiers de l'Orient. Initiation chez les compagnons du tour de France", Ethnologie française 34(3) : 517-525.

2008 Des hommes de Devoir. Les compagnons du tour de France ( $X V I I^{e}-x x^{e}$ siècle). Paris, Éditions de la Maison des sciences de l'homme («Ethnologie de la France»).

$\mathbf{2 0 1 0}$ «Le conteur, le scribe, le chansonnier. Formes et raisons de l'autobiographie chez les compagnons du tour de France", L'Homme 195-196: 193-224.

2011 "Transmettre, verbe intransitif», in Nicolas Adell et Yves Pourcher (éd.), Transmettre, quel(s) patrimoine(s)? Autour du patrimoine culturel immatériel. Paris, Michel Houdiard: 39-56.

2012 «French journeymen tradition: Convergence between French heritage traditions and UNESCO's 2003 Convention ", in Regina Bendix, Aditiya Eggert et Arnika Peselmann (éd.), Heritage Regimes and the State. Göttingen, Universitätsverlag: 177-194.

\section{Bastard, Laurent}

2008 Chefs-d'œuvre de compagnons. Sayat, De Borée.

\section{Berg, Maxine, Hudson, Pat} et Sonenscher, Michael (éd.)

1983 Manufacture in Town and Country Before the Factory. Cambridge, Cambridge University Press.

\section{Calame, François (éd.)}

1995 Charpentiers au travail. Le bois en Europe. Paris, Éditions de la Réunion des musées nationaux.

\section{Bouts de bois,}

bois debout. European Carpenters. Ateliers de Normandie.

Paris, A. Die.

\section{Cerutti, Simona}

2010 "Travail, mobilité et légitimité. Suppliques au roi dans une société d'Ancien Régime (Turin, xvIII siècle)", Annales. Histoire, Sciences sociales 3: $571-611$

\section{Connay, Jean}

1909 Le Compagnonnage, son histoire, ses mystères. Paris, Union des charpentiers de la Seine.

\section{Heinich, Nathalie} et Shapiro, Roberta (éd.)

2012 De l'artification.

Enquêtes sur le passage à l'art. Paris, Éditions de l'École des hautes études en sciences sociales.

\section{Heller-Roazen, Daniel}

2010 "Into the forge ", Qui parle: Critical Humanities and Social

Sciences 19(1): 1-8.

\section{Icher, François}

1999 Les Compagnonnages

en France au $x x^{\ominus}$ siècle.

Histoire, mémoire, représentations. Paris, Jacques Grancher.

2007 "Le chef-d'œuvre

du compagnon ", in Christian Jacob (éd.), Lieux de savoir.

I, Espaces et communautés.

Paris, Albin Michel: 246-269.

\section{Lecotté, Roger}

1980 Chefs-d'œuvre de compagnons. Paris, Chêne-Hachette.

\section{Lemaire, Marianne}

2009 Les Sillons de la souffrance. Représentations du travail en pays sénoufo (Côte d'lvoire). Paris, CNRS Editions-Editions de la Maison des sciences de l'homme ("Chemins de l'ethnologie ").

\section{Lévi-Strauss, Claude \\ 1962 La Pensée sauvage. \\ Paris, Plon ("Agora»).}

\section{Ménétra, Jacques-Louis}

1998 (1982) Journal de ma vie,

édité et présenté par Daniel Roche. Paris, Albin Michel.

\section{Thompson, Edward P.}

1988 (1963) La Formation

de la classe ouvrière anglaise. Paris, Gallimard.

\section{Trémaud, Hélène}

1962 « Documents pour l'histoire des techniques préindustrielles au musée des Arts et Traditions populaires", Documents pour l'histoire des techniques 2: 50-56.

\section{Tylor, Edward B.}

1896 Anthropology. An introduction to the study of man and civilization. New York, Appleton and Company.

\section{Varagnac, André}

1938 Définition du folklore. Paris, Société d'éditions géographiques, maritimes et coloniales.

\section{Walker, John A.}

1983 "Dream-work and art-work», Leonardo 16(2): 109-114. page 118 et ci-contre Champ de conduite de Labrie l'île d'amour, dit le Désiré, compagnon charpentier partant de Bordeaux pour aller à Paris en finissant son tour de France pour rentrer chez lui en 1826. Marseille, musée des

Civilisations de l'Europe et de la Méditerranée (MuCEM). (C) RMN Grand Palais (MuCEM)/Gérard Blot.
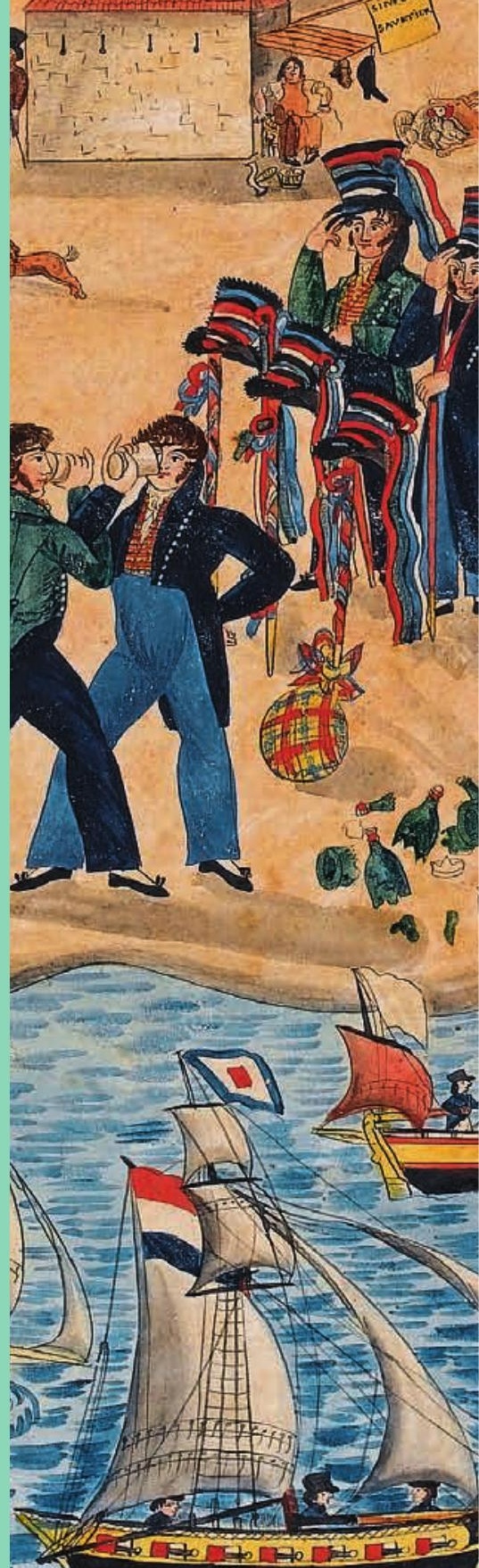


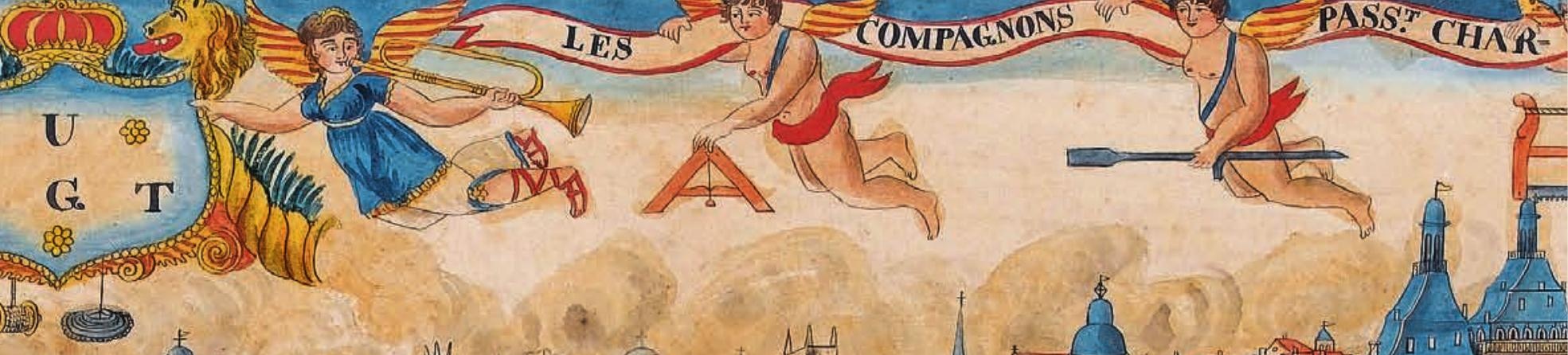
(1) (19)

की.

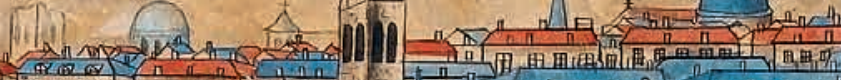
initi

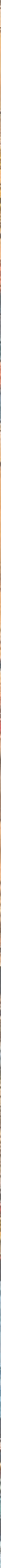

